\title{
A retrospective analysis of the epidemiology, clinical manifestations, and imaging characteristics of familial cluster- onset COVID-19
}

\author{
Kexin Huang $^{1 \#}$, Jingjing Zhang ${ }^{1 \#}$, Weidong $\mathrm{Wu}^{2}$, Di Huang ${ }^{2,3,4,5}$, Cheng $\mathrm{He}^{1,6}$, Yanli Yang ${ }^{1}$, Xianchun Zeng ${ }^{3}$, \\ Zhixia Jiang $^{4,5}$, Bangguo Li ${ }^{1}$, Heng Liu ${ }^{1}$ \\ ${ }^{1}$ Department of Radiology, Medical Imaging Center of Guizhou Province, the Affiliated Hospital of Zunyi Medical University, Zunyi, China; \\ ${ }^{2}$ Department of Hospital Infection-Control, ${ }^{3}$ Department of Radiology, ${ }^{4}$ Department of Nursing, Guizhou Jiangjunshan Hospital, Guiyang, China; \\ ${ }^{5}$ Department of Nursing, the Affiliated Hospital of Zunyi Medical University, Zunyi, China; ${ }^{6}$ Medical Imaging Department, Chongqing University \\ Central Hospital, Chongqing, China \\ Contributions: (I) Conception and design: H Liu, B Li; (II) Administrative support: W Wu, Z Jiang; (III) Provision of study materials or patients: \\ D Huang, X Zeng; (IV) Collection and assembly of data: J Zhang, Y Yang, C He; (V) Data analysis and interpretation: K Huang; (VI) Manuscript \\ writing: All authors; (VII) Final approval of manuscript: All authors. \\ \#These authors contributed equally to this work. \\ Correspondence to: Heng Liu; Bangguo Li. Department of Radiology, Medical Imaging Center of Guizhou Province, the Affiliated Hospital of Zunyi \\ Medical University, Zunyi, China. Email: zmcliuh@163.com; lbg2015@163.com.
}

Background: The coronavirus disease 2019 (COVID-19) virus has a high incidence rate and strong infectivity. The diagnosis and evaluation of familial outbreaks requires a collective consideration of epidemiological history, molecular detection methods, chest computed tomography (CT), and clinical symptoms.

Methods: A group of family patients with COVID-19 diagnosed in Guizhou, China, in February 2020, was retrospectively analyzed. As of March 1, all patients in the group have been discharged from hospital. This study tracked all patients in the group. We report the epidemiology, radiological characteristics, treatment, and clinical outcomes of these patients.

Results: We collected a group of 8 clustered cases ( 3 men and 5 women) from a family with confirmed COVID-19 infection. In the first admission diagnosis, according to the degree of clinical symptoms, the 8 patients were defined as mild type (4/8) or moderate type (4/8). They were also divided according to the CT findings into early period (1/8), progressive period (3/8), and negative on CT scan (4/8); for the first 4 patients, the corresponding CT image scores were 1, 4, 5, and 5 respectively. In this group of COVID-19 patients, half of the patients showed occult clinical manifestations and negative CT performance. We defined these patients as COVID-19-infected patients, or asymptomatic carriers.

Conclusions: The family cluster analysis indicated that COVID-19-infected patients (asymptomatic carriers) and symptomatic COVID-19 patients are distinct but coexistent. This may indicate that the infectivity and virulence of severe acute respiratory syndrome coronavirus 2 (SARS-COV-2) has decreased. In order to block the transmission pathway of this virus before it spreads, we need to identify the presence of asymptomatic carriers as early as possible.

Keywords: New coronavirus pneumonia; familial outbreaks; asymptomatic carriers; COVID-19-infected patients COVID-19 patients

Submitted Apr 03, 2020. Accepted for publication Jun 11, 2020.

doi: $10.21037 /$ atm-20-3759

View this article at: http://dx.doi.org/10.21037/atm-20-3759 


\section{Introduction}

Coronaviruses are enveloped, non-segmented, positivesense RNA viruses that appear as a corona or crown around a cell under a microscope. Coronaviruses are broadly distributed in bats, but also found in birds, cats, dogs, pigs, whales, and humans. At present, seven known coronaviruses can infect humans.

At the end of 2019, corona virus disease-2019 (COVID-19) broke out in Wuhan, Hubei, China, and, as of early March 2020, more than 90,000 people have been diagnosed with COVID-19 worldwide (1). The International Virus Classification Commission officially named the virus that caused the disease severe acute respiratory syndrome coronavirus 2 (SARS-CoV-2). SARS$\mathrm{CoV}-2$ infects the human endothelium by binding its spike protein, that has the angiotensin 2 receptor, onto human cells (2). The virus can cause serious lung inflammation and damage the nervous system and digestive tract.

Many studies have confirmed the importance of realtime polymerase chain reaction (RT-PCR) in the diagnosis, treatment evaluation, and follow-up of COVID-19 infection, and chest computed tomography (CT) is an important complement to nucleic acid testing $(3,4)$. While studies have generated data on the epidemiology, clinical symptoms, and CT findings of COVID-19 patients, the comprehensive analysis of patients with familial clustering has not yet been undertaken. In order to improve the diagnosis and treatment of patients with familial cluster outbreak, we retrospectively analyzed the medical records and imaging data of a group of COVID-19 patients with familial aggregation.

We present the following article in accordance with the STROBE reporting checklist (available at http://dx.doi. org/10.21037/atm-20-3759).

\section{Methods}

This study was approved by the medical ethics committee and institutional review board of the Guizhou Jiangjunshan Hospital. The study was conducted in accordance with the Declaration of Helsinki.

We collected the data of a group of eight clustered cases from a family with confirmed COVID-19 infection in February 2020 in Guizhou province. RT-PCR assay confirmation was performed using test kits manufactured by Da An Gene Co (Guangzhou, China). Laboratory confirmation of the virus was performed using RT-qPCR.
The RT-qPCR laboratory confirmation was started on the second day after the patient performed one week of ET. If the laboratory test remained positive, then the test was repeated every 48 hours until the test was negative. The collection of RT-qPCR data was performed uniformly by the technicians of General Mountain Hospital of Guizhou Province. The technician was strictly trained to ensure the accuracy of the dae to negative RT-qPCR of the patient was used as the primary outcome. Nasopharyngeal swab specimens were collected from all patients, and the SARSCov-2 RNA was tested by real-time polymerase chain reaction (RT-PCR) within tta.We classified COVID-19 into mild, moderate, severe, and critical clinical types according to the degree of clinical symptoms. According to the CT findings, the patients were divided into four periods: early, progressive, transitional, and diffuse critical type.

The study followed 13 people in a family with a history of close contact. Six of them lived in Wuhan for a long period, while the other members had settled in Guizhou. They had not recently been exposed to wild animals, fever/ pneumonia patients, seafood markets, or local hospitals. On January 16, 2020, a male family member took a train to Guizhou. His wife stayed in Wuhan and never left. Upon his arrival in Guiyang, the man had close contact with the family members living there. On January 22, 2020, 4 other family members drove to Guiyang from Wuhan and were immediately reunited with the first man. We observed and RT-PCR tested all the close contacts of the family since January 16. In total, eight COVID-19 patients were confirmed and admitted to hospital consecutively. During hospitalization, we classified the patients according to their clinical symptoms, closely followed their clinical symptoms, reviewed chest CT images, and rechecked nucleic acid throat swabs.

To discharge suspected COVID-19-infected patients, the criteria set out in the guidelines of the Diagnosis and Treatment of Pneumonitis Caused by 2019-nCoV (trial sixth version) published by the China government (5), must first be met. After discharge, patients were quarantined for 14 days and regularly checked by RT-PCR. As of March 1, all 8 people in this group have been discharged.

UCT 510 (United-Imaging; Shanghai, China) was used in all CT scans. The scans were performed from the upper thoracic inlet to the inferior level of the costophrenic angle in the supine position. The scanning parameters were set as follows: $120 \mathrm{kVp}$ tube voltage, automatic tube current modulation, a $512 \times 512$ matrix, $1.5-5 \mathrm{~mm}$ slice thickness, a $313 \mathrm{~mm} \times 313 \mathrm{~mm}$ field of view, $1.5-8 \mathrm{~mm}$ spacing between 


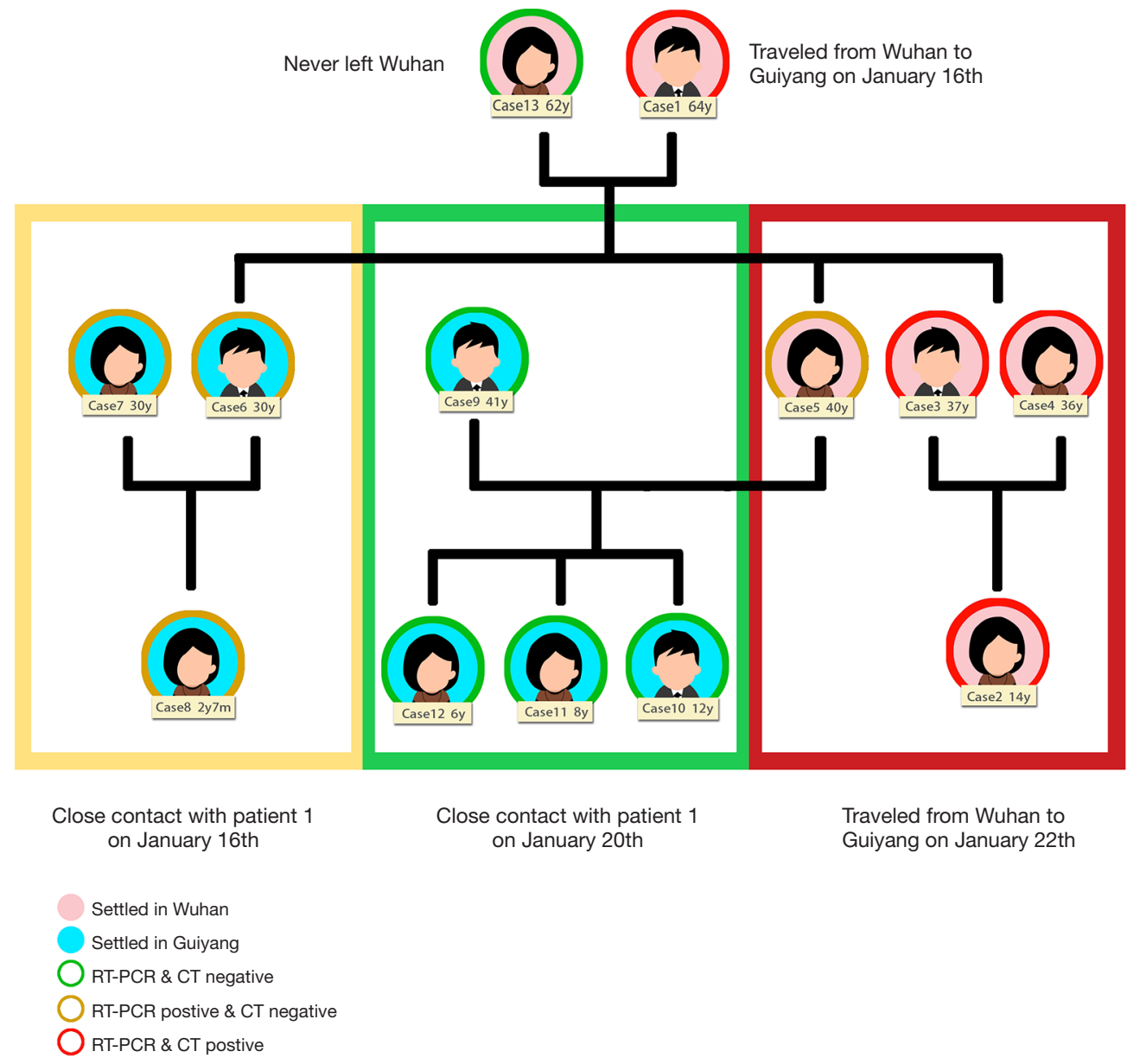

Figure 1 The relationship and demographic information of case 1 to case 13, numbered according to the order of the onset are shown.

slices, window center at -400 , and a window width of 1,400 . All images were reconstructed in increments using a slice thickness of $1.5 \mathrm{~mm}$.

Informed consents were obtained from the patients.

\section{Image analysis}

Two senior chest radiologists independently analyzed all images. Image analysis focused on the lesion features of each patient, including distribution characteristics, number of lobes involved, lobe of lesion distribution, and patterns of the lesion; the patterns included ground glass opacification (GGO), consolidation, interlobular septal thickening, air bronchogram sign, adjacent pleura thickening, pleural effusion, and thoracic lymphadenopathy. The alterations caused by underlying lung diseases, such as bronchiectasis or cancer, were not included in this study. The two doctors comprehensively analyzed the image information to determine the staging and scoring of the chest CT. When differences of opinion occurred, a consensus was reached through discussion.

\section{Results}

In total, there were 13 cases in our study from 1 family unit. We defined the case numbers from 1 to 13 according to the order of the onset. A total of 8 members of the family, cases 1-8, were confirmed positive for COVID-19 by RT-PCR (3 males and 5 females). Their relationship and demographic information is shown in Figure 1.

Our study began at the time of symptom onset and ended at the time of discharge. For asymptomatic patients, the starting time was when the first RT-PCR test was positive. The longest duration of observation was 24 days, and the shortest duration was 15 days, averaging 20.8 days (Figure 2). The earliest clinical symptoms appeared in the family 


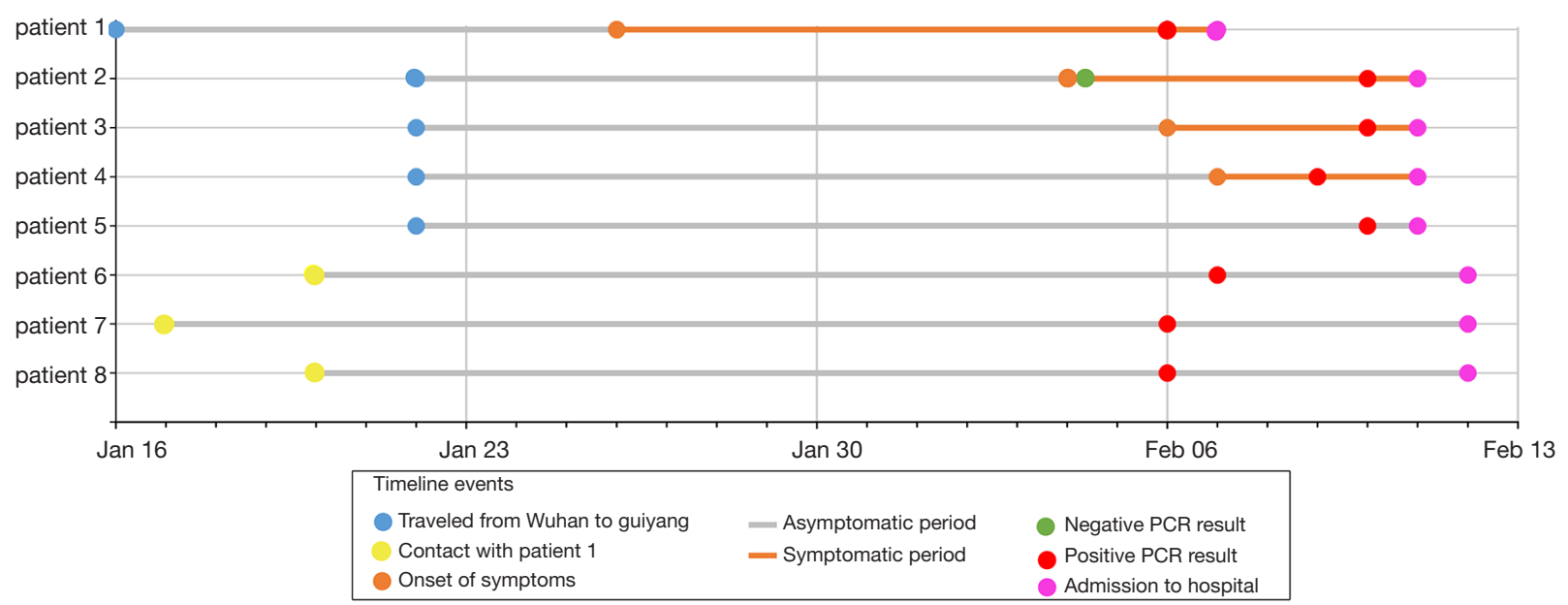

Figure 2 Time line of exposure to the asymptomatic carrier of the novel coronavirus that caused COVID-19 in a familial cluster.

members who returned to Guizhou from Wuhan first. A few days later, CT changes and clinical manifestations with different degrees of viral pneumonia infection were observed in cases $2-4$ who had never had lung disease. The youngest age was 14 years with an average age of 37.8 , and all had a basic medical history; 3 had fatty liver and 1 had hyperuricemia. Case 5, who had underlying lung disease and a history of breast cancer, exhibited no signs of viral infection on the chest CT, and cases 6-8 did not show significant clinical symptoms during the observation period and were negative on CT scan. The 8 patients were finally diagnosed with COVID-19 by RT-PCR and admitted to the hospital.

In the first admission diagnosis, cases $1-4$ were judged as a moderate type. The 4 patients were divided into the early period (1 case) and progressive period (3 cases); the corresponding CT image scores were $1,4,5$, and 5 respectively (6). The remaining 4 patients were designated as mild types (Figure 3, Table 1). During the period of hospitalization, most of the patients (4/8) presented with cough symptoms, while the rest presented with sputum (1/8), fever (2/8), chills (1/8), and muscle soreness (1/8). These symptoms were obviously relieved or disappeared about 1 week before discharge.

The CT imaging suggested that during hospitalization all patients except 1 were in mild and stable condition. The CT score showed that lung lesions were most serious about 10 days after the onset of clinical symptoms. In the 4 patients with abnormal chest CT change, 1 lesion was located in the upper lobe of the right lung, and 3 were located in the lower lobe of both lungs. There were various near-complete resolutions of pulmonary opacities, groundglass density shadows, solid components, and air-bronchial meteorology seen in some lesions. In the transition period, bilateral GGO and linear consolidation was observed. No pleural effusion or lymphadenopathy was found among the cases (Figure 4).

\section{Discussion}

\section{Epidemiology}

From the end of 2019 to the beginning of 2020, the arrival of the Spring Festival increased opportunities for people to gather, and, coupled with the high incidence of COVID-19, group cases rapidly emerged.

Epidemiological follow-up of this group of patients revealed that patient 1 was infected with COVID-19 on the way back to Guiyang from Wuhan, and then quickly spread the disease among family members. The source of infection is clear and the infected people cover all age stages. In total, 8 of the 13 people were diagnosed with COVID-19, 50\% of the patients had negative CT, and the clinical symptoms were mild (Table 1). In the early period of the disease, the diagnosis of these patients could only rely on RT-PCR testing to confirm diagnosis.

Some patients in this group were likely to have been infected during the incubation period. Among the infected family members, there were four asymptomatic infected members. This suggests that the incubation period is contagious. The infectious ability of symptomatic patients is unclear in the incubation period. 


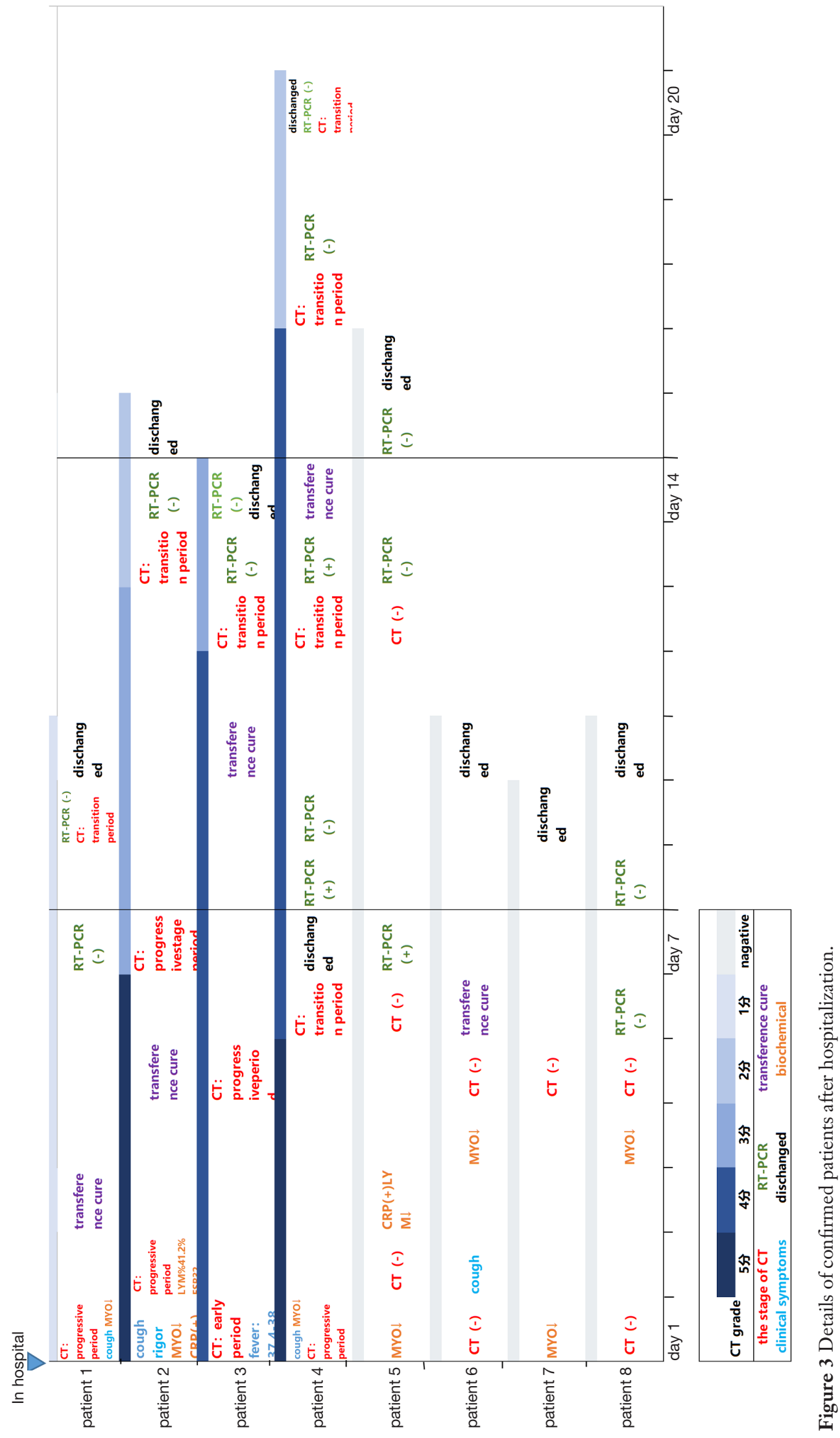


Table 1 Criteria for clinical severity of COVID-19

\begin{tabular}{|c|c|}
\hline Types & Findings \\
\hline Moderate & Fever, respiratory symptoms, imaging findings of pneumonia \\
\hline \multirow[t]{3}{*}{ Severe } & Meet any of the followings: \\
\hline & $\mathrm{PaO}_{2} / \mathrm{FiO}_{2}<300 \mathrm{mmHg}$ \\
\hline & $\begin{array}{l}\text { *Patients showing a rapid progression ( }>50 \% \text { ) on CT imaging within } 24-48 \text { hours should be managed as severe (added in } \\
\text { the trial sixth edition) }\end{array}$ \\
\hline \multirow[t]{2}{*}{ Critical } & Meet any of the followings: \\
\hline & "Extra pulmonary" organ failure, intensive care units needed \\
\hline
\end{tabular}

The content represented by "*” is data from (5). $\mathrm{RR}$, respiratory rate; $\mathrm{SpO}_{2}$, oxygen saturation; $\mathrm{PaO}_{2}$, partial pressure of oxygen; FiO fraction of inspired oxygen.

\section{Clinical symptoms and diagnosis}

Dry cough was the most common clinical symptom observed in this group. Previous studies have shown that fever, cough, and dyspnea are the most common symptoms of lower respiratory tract infection (7). In this group of confirmed patients, no significant upper respiratory tract infection symptoms were observed. These symptoms fit with the suspicion that the cells targeted by the virus might be located in the lower airway. More accurate detection of lower respiratory tract specimens was also emphasized in the latest infectious pneumonia protocol (trial version 7), released on March 4, 2020.

However, lower respiratory tract nucleic acid testing is not yet widely available, and, due to limitations of sample collection and kit performance, the total positive rate of RT-PCR for throat swab samples was reported to be about $30-60 \%$ at initial presentation (8). Chest CT, as the best supplement for pneumonia diagnosis, is relatively easy to perform and can produce a fast diagnosis. However, it is worth noting that $50 \%$ of the patients in this group had a negative CT diagnosis. Moreover, patients with no symptoms or only mild clinical symptoms and negative CT findings already have the ability to infect others (9). There was a positive result on the RT-PCR test but no abnormalities in the chest CT in these COVID-19- infected persons.

Because of the obvious clinical manifestations and CT changes, it is easy to treat some COVID-19 patients, but it is the undetected COVID-19-infected individuals that we should pay more attention to. Therefore, the importance of nucleic acid testing and CT in the diagnosis and evaluation of COVID-19 should not be underestimated, and relying solely on a single technique for clinical diagnosis should be avoided (6).

\section{RT-PCR detection}

During hospitalization of case 4 , we noted a dynamic conversion of RT-PCR results (from negative to positive, and then from positive to negative). We assume that the negative test result arose due to error during the course of the disease. It might have been the result of collecting a sample with a low viral load, unreliable testing agents, or because of other reasons. As mentioned above, COVID-19 patients mostly have lower respiratory symptoms, but because throat swabs are easier to sample, they are used in most studies and in our hospital. Doctors should consider that the sampling source (upper or lower respiratory tract) may affect the RT-PCR testing results. Similarly, postcureisolated COVID-19 patients who had met the discharge criteria were also reported positive by RT-PCR after 

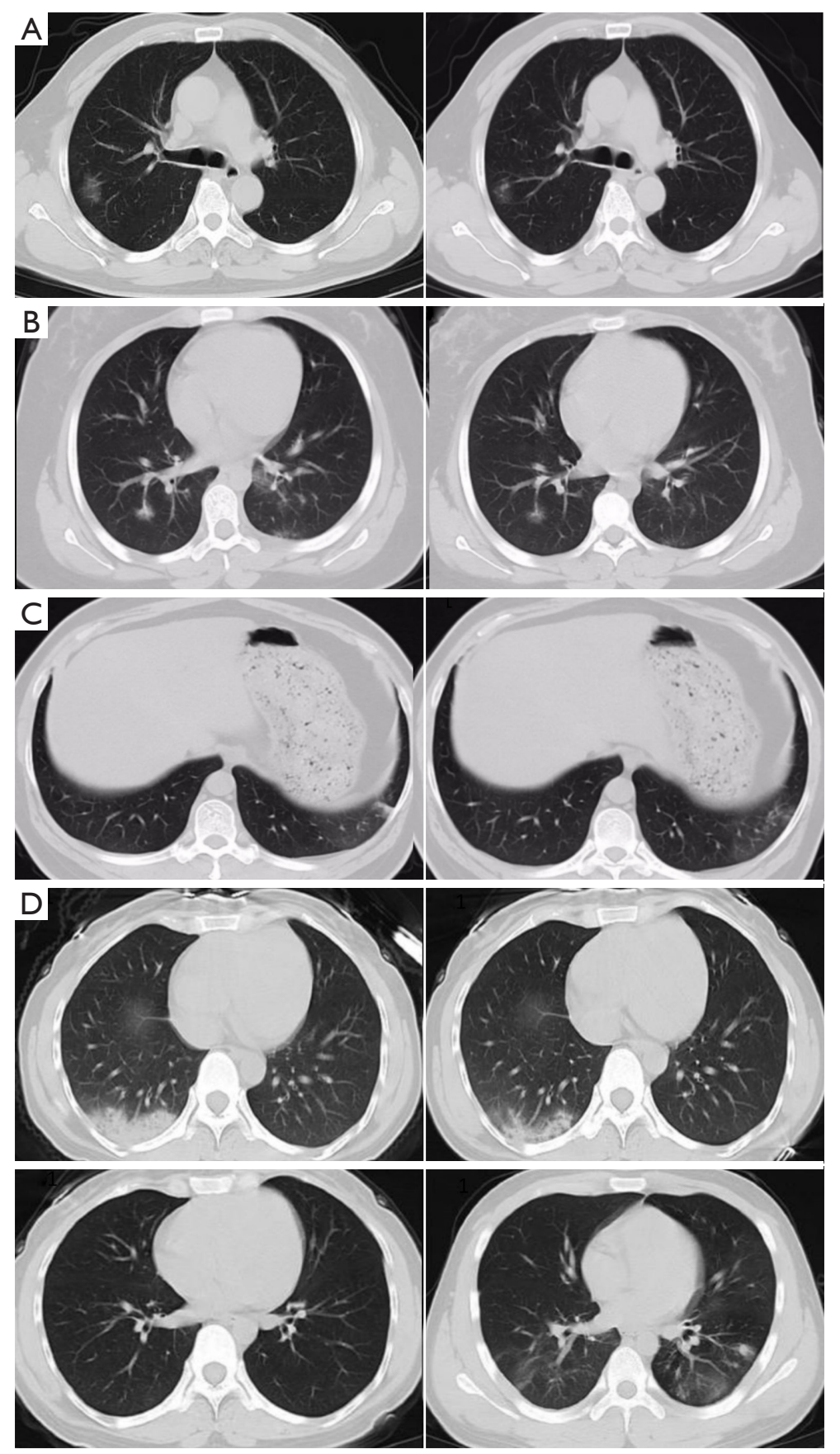

Figure 4 CT images of the patients. (A) Patient 1. On February 7, CT showed pure ground-glass opacity in the subpleural area of the upper lobe of the right lung. On February 15, the lesion was narrowed and the density decreased. (B) Patient 2. On February 16, CT showed that the right lower lobe of the lung, solitary grounded ground-glass opacity. On February 22, a reduction in the lesion area and density at the same level. (C) Patient 3. On February 12, CT showed that the left lower lobe of the lung had pure consolidation lesions near the pleural area. On February 22, the density of the lesion decreased in the same layer. (D) Patient 4. On February 12, CT showed that the upper pleura of the lower lobe of the left lung had a dense, patchy, ground-glass opacity with fan-shaped consolidation. The second scan on February 16 showed that the same level of disease-density decreased at the edge mold and the edge of the halo sign. The third examination on February 22 showed that the lesions were basically absorbed within the visual field, and no definite lesions were found. On March 1, before discharge from the hospital, CT showed bilateral ground-glass opacities and linear consolidation. 
discharge. More research is needed to understand the cause of this (10).

\section{CT scan of the chest}

The CT results demonstrate that multiple re-examinations of chest CT can accurately reflect the disease evolution and monitor the treatment effect. There were some differences between the staging and scoring results of CT in this group. This may be because in the critical phase (critical illness) (11), chest CT is usually associated with specific imaging findings. However, most of the chest CT findings in this group were in the early, progressive, or transitional period (11).

According to Pan's methodology (12), a semiquantitative scoring system was used to estimate the pulmonary involvement of all these abnormalities quantitatively based on the area involved. The change in score was more sensitive to the increase or decrease of chest lesions, which helped the continuous monitoring of the changes in the disease. However, in severe or critically ill patients, when considering the severity of lung lesions, the observation indicators are complex and evaluation requires experience. Whether CT changes in these patients can still be quantified requires further research.

The chest CT findings of COVID-19 were consistent with recent literature, and many characteristic changes were seen. In the early period of the disease, atypical thin patchy ground glass density shadows and localized patchy masses were found. In the progressive period, multifocal bilateral GGOs with patchy consolidations and prominent peripherally subpleural distributions were evident and had a posterior or lower lobe predilection. In some of the lesions, bronchial meteorology could be seen. In the transition period, the density and range of the lesions decreased and the consolidation lesions of the lung were gradually absorbed and dissipated. Part of the lesion transformed into a residual proliferative streak. These strands may indicate the development of fibrosis. However, since COVID-19 progression has not been fully explored and the destruction of alveolar basal cells cannot be seen pathologically, we cannot directly classify these proliferative streaks as irreversible fibrosis (13). In this group of patients shows typical clinical and imaging findings of COVID-19. Identification of other viral pneumonia such as H7N9, SARS and MERS. Those diseases showed typical clinical as fever and chill, the COVID-19patients could also had muscle ache, confusion and headache. Imaging findings of various viral pneumonia were similar: lower lobe involved, interlobular septal and intralobular septal, extensive GGO and occasional septal thickening and pleural. Severe group of patients shows diffuse heterogeneous consolidation with GGO. In summary, the diagnosis of COVID-19 should combine epidemiological history, clinical and imaging manifestations, RT-PCR test and depends on pathogen detection.

In this group of patients with familial cluster onset, the epidemiology was clear, the disease course cycle unified, and the CT manifestations conformed to the disease course. Half of the patients in this group had asymptomatic COVID-19 infection. This may indicate that the infectivity and virus virulence of the patients during the incubation period decreased. This comprehensive study of patients with a family cluster might be able to provide clues as to whether the virus is changing, adapting, or hiding in the process of continuous transmission while maintaining certain disease characteristics.

Furthermore, the study suggests that the patients can be divided into two distinct groups according to clinical symptoms: COVID-19-infected patients and COVID-19 patients. We need to identify the presence of COVID-19infected patients as early as possible by using a combination of epidemiological history, RT-PCR, chest CT, and clinical symptoms, in order to block the transmission pathway before it spreads.

Some limitations to our study should also be addressed. First, this group of patients had only a limited number of cases with most being mild in severity. We thus lack data on severe patients, making it difficult to use multivariate adjustment methods to assess the host response to disease severity or find correlations between variables. A second limitation is that this is a small-scale family analysis; gathering more family-aggregated models and standardizing the data will lead to more convincing evidence for further research.

\section{Conclusions}

This study shows that COVID-19 patients in the incubation period and COVID-19-infected patients (asymptomatic carriers) are still able to infect others. This indicates that the epidemiology of the disease can pass undetected and spread without the proper detection methods. This information will be useful for clinicians in detecting infected patients and monitoring the population to control the spread of the virus, even in the absence of obvious symptoms of infection. 


\section{Acknowledgments}

Funding: None.

\section{Footnote}

Reporting Checklist: The authors have completed the STROBE reporting checklist. Available at http://dx.doi. org/10.21037/atm-20-3759

Data Sharing Statement: Available at http://dx.doi. org/10.21037/atm-20-3759

Conflicts of Interest: All authors have completed the ICMJE uniform disclosure form (available at http://dx.doi. org/10.21037/atm-20-3759). The authors have no conflicts of interest to declare.

Ethical Statement: The authors are accountable for all aspects of the work in ensuring that questions related to the accuracy or integrity of any part of the work are appropriately investigated and resolved. This study was approved by the medical ethics committee and institutional review board of the Guizhou Jiangjunshan Hospital. The study was conducted in accordance with the Declaration of Helsinki (as revised in 2013). Informed consents were obtained from the patients.

Open Access Statement: This is an Open Access article distributed in accordance with the Creative Commons Attribution-NonCommercial-NoDerivs 4.0 International License (CC BY-NC-ND 4.0), which permits the noncommercial replication and distribution of the article with the strict proviso that no changes or edits are made and the original work is properly cited (including links to both the formal publication through the relevant DOI and the license). See: https://creativecommons.org/licenses/by-nc-nd/4.0/.

\section{References}

1. Chan JF, Yuan S, Kok KH, et al. A familial cluster of pneumonia associated with the 2019 novel coronavirus indicating person-to-person transmission: a study of a family cluster. Lancet 2020;395:514-23.

2. Xu X, Chen P, Wang J, et al. Evolution of the novel coronavirus from the ongoing Wuhan outbreak and modeling of its spike protein for risk of human transmission. Sci China Life Sci 2020;63:457-60.
3. Corman VM, Landt O, Kaiser M, et al. Detection of 2019 novel coronavirus (2019-nCoV) by real-time RTPCR. Euro Surveill 2020. doi: 10.2807/1560-7917. ES.2020.25.3.2000045.

4. Wei-jie Guan, Zheng-yi Ni ,Yu Hu, et al. Clinical Characteristics of Coronavirus Disease 2019 in China.. N Engl J Med 2020. [Epub ahead of print].

5. General Office of National Health Committee. Notice on the issuance of a program for the diagnosis and treatment of novel coronavirus (2019-nCoV) infected pneumonia (trial sixth edition) (2020-02-18). Available online: http://www. nhc.gov.cn/yzygj/s7653p/202002/8334a8326dd94d329df35 1d7da8aefc2.shtml? from=timeline (accessed Feb 24, 2020).

6. Pan F, Ye T, Sun P, et al. Time Course of Lung Changes On Chest CT During Recovery From 2019 Novel Coronavirus (COVID-19) Pneumonia. Radiology 2020. [Epub ahead of print].

7. Chen N, Zhou M, Dong X, et al. Epidemiological and clinical characteristics of 99 cases of 2019 novel coronavirus pneumonia in Wuhan, China: a descriptive study. Lancet 2020;395:507-13.

8. Yang Y, Yang M, Shen C, et al. Evaluating the accuracy of different respiratory specimens in the laboratory diagnosis and monitoring the viral shedding of $2019-\mathrm{nCoV}$ infections. medRxiv 2020. doi: http://doi.org/10.1101/202 0.02.11.20021493.

9. Chung M, Bernheim A, Mei X, et al. CT Imaging Features of 2019 Novel Coronavirus (2019-nCoV). Radiology 2020;295:202-7.

10. Wang YXJ, Liu WH, Yang M, et al. The role of CT for Covid-19 patient's management remains poorly defined. Ann Transl Med 2020;8:145.

11. Huang C, Wang Y, Li X, et al. Clinical features of patients infected with 2019 novel coronavirus in Wuhan, China. Lancet 2020;395:497-506.

12. Chang YC, Yu CJ, Chang SC, et al. Pulmonary sequelae in convalescent patients after severe acute respiratory syndrome: evaluation with thin-section CT. Radiology 2005;236:1067-75.

13. Strieter RM, Mehrad B. New mechanisms of pulmonary fibrosis. Chest 2009;136:1364-70.

Cite this article as: Huang $\mathrm{K}$, Zhang $\mathrm{J}$, Wu W, Huang $\mathrm{D}$, He C, Yang Y, Zeng X, Jiang Z, Li B, Liu H. A retrospective analysis of the epidemiology, clinical manifestations, and imaging characteristics of familial cluster-onset COVID-19. Ann Trans Med 2020;8(12):747. doi: 10.21037/atm-20-3759 